\title{
Concomitant Intraspinal and Retroperitoneal Hemorrhage Caused by an Aneurysm on the Celiac Artery: A Case Report
}

\author{
Katrien Vermeulen ${ }^{1}$ Veerle Schwagten ${ }^{1}$ Tomas Menovsky ${ }^{2}$ \\ ${ }^{1}$ Department of Emergency Medicine, University Hospital, Antwerp, \\ Belgium \\ 2 Department of Neurosurgery, University Hospital, Antwerp, Belgium \\ Address for correspondence Katrien Vermeulen, RL, MD, Department \\ of Emergency Medicine, University Hospital, Wilrijkstraat 10, 2650 \\ Edegem, Antwerp, Belgium (e-mail: kvermeulen@gmail.com).
}

J Neurol Surg Rep 2015;76:e28-e31.

\begin{abstract}
Spontaneous spinal hemorrhage is a rare condition. We present a case in which the diagnosis was complicated by a concomitant intra-abdominal hemorrhage. The patient, taking coumarins, presented with acute back pain and abdominal pain and progressive paresis of the lower limbs. Computed tomography angiography of the abdomen showed an intra-abdominal hemorrhage and an aneurysm of the celiac trunk. MR (magnetic resonance) imaging of the spine revealed a combined subdural and epidural

Keywords

- spinal hemorrhage

- coumarins

- celiac trunk aneurysm

- paresis

- abdominal hemorrhage hemorrhage from $\mathrm{C} 1$ to L1. Both sites were treated conservatively. After 6 months the patient regained strength in both legs with some persistent loss of strength in the left leg. Follow-up MR imaging showed complete resolution of the spinal hemorrhage. The celiac artery aneurysm was treated conservatively. We suggest that the rupture of the celiac artery aneurysm caused increased intra-abdominal pressure leading to spinal hemorrhage. Emergency staff should be aware of the possibility of two rare but concomitant conditions.
\end{abstract}

\section{Introduction}

Spontaneous spinal hemorrhage is a rare, but important emergency. Epidural as well as subdural and subarachnoid hemorrhage can occur. In most cases, no etiology can be identified. These idiopathic hemorrhages represent around $40 \%$ of cases. The use of anticoagulant therapy is the most important etiologic factor. Other predisposing conditions include hypertension, increased abdominal pressure (leading to increased venous pressure of the spinal plexus), coagulopathies, and vascular malformation. In unusual cases, the diagnosis can be challenging due to concurrent pathologies that draw attention away from the spinal hemorrhage.

We present a case of a 61-year-old male patient with abdominal pain due to ruptured celiac trunk aneurysm and a progressive sensorimotor deficit of the legs.

received

June 6, 2014

accepted after revision

August 6, 2014

published online

January 16, 2015

\section{Case Report}

A 61-year-old male patient was transferred to our hospital with lower back pain and acute onset sensorimotor paresis of the lower limbs. The night before admission, the patient suffered from diffuse abdominal pain with vomiting. In the morning the patient experienced numbness in the left leg and he was unable to walk and stand on the left leg. His medical history included placement of a mechanical bileaflet aortic valve 11 years ago for which the patient was on Acenocoumarol.

On admission the patient was pale with generalized marbled skin. Blood pressure was $95 / 65 \mathrm{~mm} \mathrm{Hg}$, pulse 90 beats per minute. The abdomen was tense and slightly painful on palpation. The femoral pulses were symmetric but weak. Neurologic evaluation showed numbness of the lower limbs. A sensible cut was present at the level of the umbilicus. There

\footnotetext{
(c) 2015 Georg Thieme Verlag KG Stuttgart · New York
}

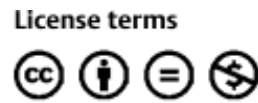




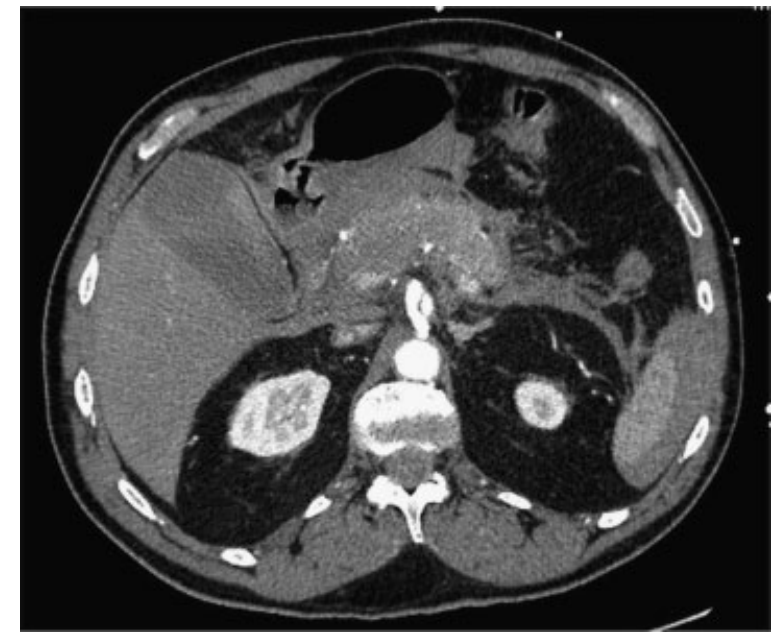

Fig. 1 CT scan which shows the dissection in the aneurysm at the celiac trunk and the surrounding intra-abdominal hemorrhage. CT, computed tomography.

was a near complete paralysis of both legs with loss of reflexes. However, a normal perianal sensibility and normal anal sphincter tone were present.

Laboratory investigations showed a hemoglobin level of $11.8 \mathrm{mg} / \mathrm{dL}$ and prothrombin time of $11 \%$ (international normalized ratio of 5.5) and an activated partial thromboplastin time of 48.3 seconds.

Computed tomography (CT) angiography showed an intraabdominal, retroperitoneal hematoma with a stenotic celiac trunk and a poststenotic saccular aneurysm (-Figs. $\mathbf{1}$ and $\mathbf{2}$ ). The probable source of the hemorrhage was small mesenteric branches. An angiography of the aorta and its branches revealed no active hemorrhage ( - Fig. 3 ). A competent artery of Adamkiewicz was localized at T11 on the left side. Stenosis of the celiac trunk with an aneurysm distally was confirmed. An urgent magnetic resonance imaging (MRI) of the spine showed an intraspinal, intradural hemorrhage, extending from the $\mathrm{C} 1$ to $\mathrm{L} 1$. There was also an epidural component of

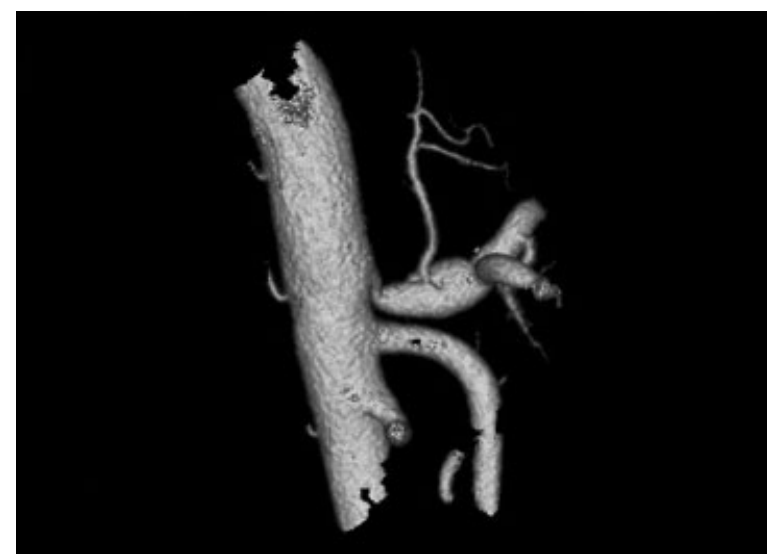

Fig. 2 3D-reconstruction image of the aneurysm. 3D, three-dimensional.

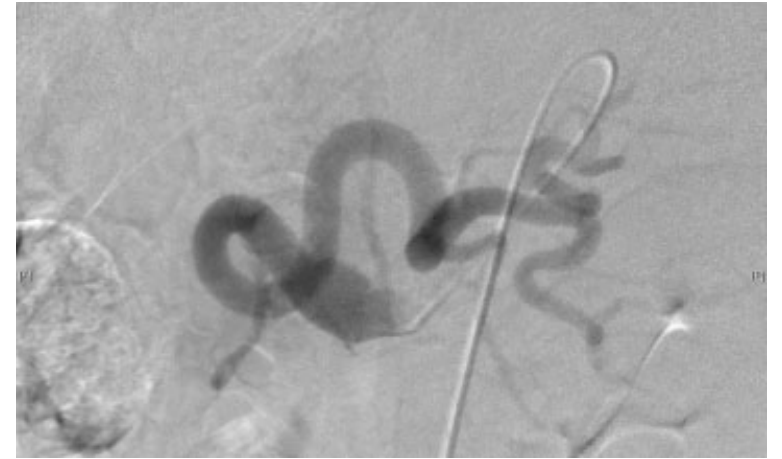

Fig. 3 Angiographic image of the aneurysm. There is no "blush," no active hemorrhage, seen on the image.

blood. Compression of the spinal cord at the midthoracic level was observed (-Fig. 4).

The patient was admitted to the intensive care unit (ICU) and pressure control with a calcium antagonist and betablocking agents. Vitamin $\mathrm{K}$ as well as prothrombin complex concentrate were administered to correct abnormal hemostasis.

Concerning both hemorrhages, a conservative treatment was considered the best option for the patient. The intraspinal hemorrhage was mostly located anterior and over a long distance in the vertebral column, so posterior decompression was not indicated. Because there was no active hemorrhage

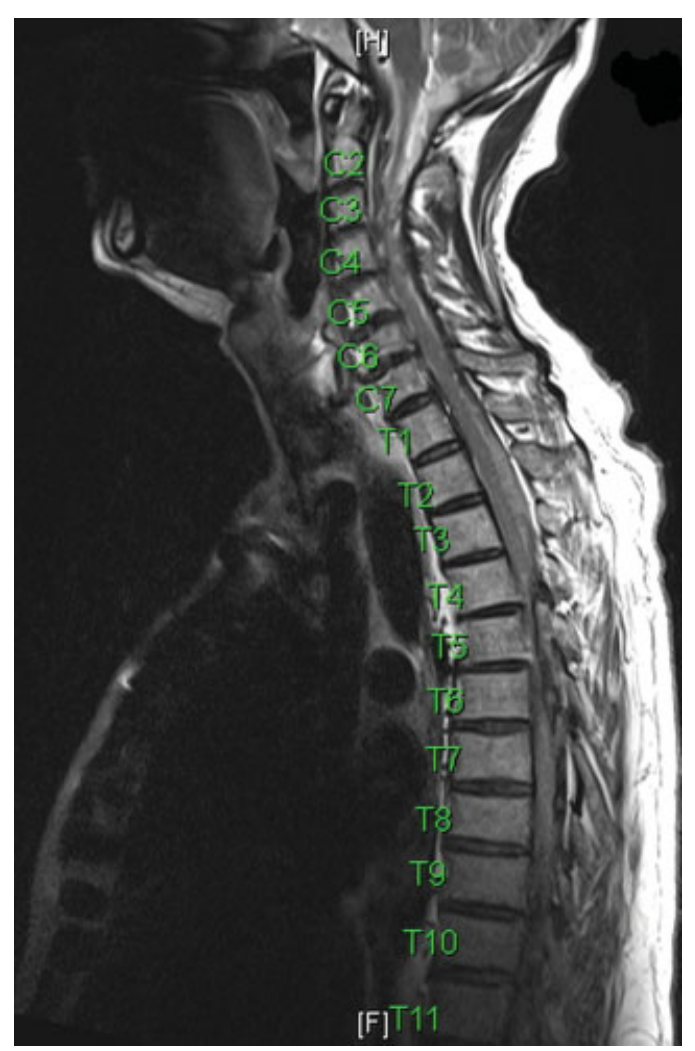

Fig. 4 MRI image of the thoracic spine showing intraspinal hemorrhage. $\mathrm{MRI}$, magnetic resonance imaging. 
from the celiac trunk aneurysm, conservative treatment was decided.

Follow-up CT angiography of the abdomen 14 days after admission showed a stable aneurysm with a diameter of $1.5 \mathrm{~cm}$ and no further active hemorrhage. The stay at the ICU was complicated by a deep venous thrombosis despite therapeutic doses of low-molecular-weight heparin. Compression therapy of the lower limbs was started. After 18 days, the patient could be transferred to the neurosurgical ward. The strength in the right leg was recovering with persistent loss of strength and paresthesia in the left leg.

CT angiography of the abdomen was repeated after 6 months and showed no increase of the aneurysm.

Follow-up at 12 months showed improved motor function. There is a complete recovery of the right leg and persistent paresis (grade 4) of the left leg. The patient is self-sufficient and walks with a cane. There is limited urinary retention with need of self-catheterization two times a day. MRI shows resolution of the hematoma and the presence of arachnoiditis.

Concerning his celiac trunk aneurysms the patient has no abdominal complaints. CT scanning of the abdomen shows resolution of the hematoma and an unchanged aneurysm.

\section{Discussion}

Spontaneous spinal hemorrhage is a rare condition. Epidural as well as subdural and subarachnoid hemorrhage can occur. Most commonly hemorrhage occurs in the sixth decade, although it can occur in any age group. In most cases no etiology can be identified. These idiopathic hemorrhages represent $40 \%$ of cases. ${ }^{1,2}$ Second, coagulopathies account for $17 \%$ as an etiological factor for spinal hematomas. ${ }^{2}$ There are other predisposing conditions such as hypertension and increased abdominal pressure. The most common region of bleeding is the cervicothoracic and thoracolumbar region. ${ }^{2}$ These hemorrhages are mostly situated in the posterior or posterolateral region of the spine where the posterior internal vertebral venous plexus is located. ${ }^{1,3,4}$ The vertebral venous system is a valve less anatomical system which is very susceptible to congestion. Blood flow is dependent on hydrostatic factors and the thoracoabdominal pressure. The Batson venous plexus (Batson veins) is a network of valve less veins that connect the deep pelvic veins and thoracic veins to the internal vertebral venous plexuses. ${ }^{3,4}$ Any elevation in the abdominal or thoracic pressure can be transferred into the epidural venous plexus causing distention of the veins and subsequent hemorrhage. Clinically a spinal hemorrhage usually presents with intense, sharp back pain, located around the level of the hemorrhage. This pain can be followed by progressive paralysis below the affected spinal level. ${ }^{2,5}$

Visceral aneurysm is also a rare condition with an incidence in postmortem studies ranging from 0.01 to $0.2 \%$. There is a high mortality rate of around $70 \%$ in case of rupture. ${ }^{6}$ The most common location of a visceral aneurysm is the splenic artery (60\%), followed by the hepatic artery (20\%). Only in $4 \%$ the origin of the aneurysm is on the celiac trunk. ${ }^{6}$ The pathophysiology is not completely understood, but atherosclerosis seems to play an important but not solely role in the etiology. Congenital arterial wall malformation (Marfan, Ehlers-Danlos) and inflammatory disease (Still disease, systemic lupus erythematosus) are predisposing factors for formation of visceral aneurysms. ${ }^{6,7}$ The risk of rupture is diameter related. There is a general consensus to treat visceral aneurysms larger than $2 \mathrm{~cm}$ and the symptomatic ones regardless their size. ${ }^{7}$ If treated, this can be done surgically or endovascularly depending on various factors.

This patient suffered from a hemorrhagic aneurysm on the celiac trunk with a large intra-abdominal hematoma. We hypothesized that this hematoma caused an elevated retroperitoneal pressure, leading to elevated pressure in the epidural spinal venous plexus. The use of coumarins facilitated spinal hemorrhage. The pain was initially localized abdominally (probably due to the rupture of the aneurysm) and a few hours later also a sharp back pain developed (probably due to the spinal hematoma). This was followed by progressive paralysis of the lower limbs. The diagnosis of the spinal hemorrhage was postponed because of the already detected celiac trunk aneurysm on abdominal CT scan. A subsequent MRI, performed because of progressive sensorimotor symptoms of lower extremities, revealed the intraspinal hemorrhage from the craniocervical region to the lumbar region mainly on the anterior side of the myelum. Most probably, the hemorrhage was so extensive due to the hypocoagulable state.

The intra-abdominal hemorrhage in our patient did not originate from the aneurysm, but from small mesenteric branches. So the aneurysm was considered stable and conservative treatment with regular follow-up was planned.

Surgical treatment of the spinal hematoma was not performed for two important reasons. First, adequate removal of the hematoma would require an extensive laminectomy adding significant morbidity to the procedure. Second, prone position (required for laminectomy) was contraindicated because of the retroperitoneal hematoma and added elevated venous pressure.

Despite conservative treatment, including optimizing the coagulation status, the patient recovered relatively well.

With this case report, we want to draw attention to the difficulty of obtaining an accurate diagnosis. Emergency staff should be aware of the possibility of two rare but concomitant conditions. Multidisciplinary consulting should be the key in diagnosis and treatment strategy in patients with multiple life-threatening pathologies.

Extensive search of literature did not reveal any similar cases as described in this article.

\section{References}

1 Liu Z, Jiao Q Xu J, Wang X, Li S, You C. Spontaneous spinal epidural hematoma: analysis of 23 cases. Surg Neurol 2008;69(3):253-260, discussion 260

2 Kreppel D, Antoniadis G, Seeling W. Spinal hematoma: a literature survey with meta-analysis of 613 patients. Neurosurg Rev 2003; 26(1):1-49

3 Taniguchi LU, Pahl FH, Lúcio JE, et al. Complete motor recovery after acute paraparesis caused by spontaneous spinal epidural hematoma: case report. BMC Emerg Med 2011;11:10 
4 Messerer M, Dubourg J, Diabira S, Robert T, Hamlat A. Spinal epidural hematoma: not always an obvious diagnosis. Eur J Emerg Med 2012;19(1):2-8

5 Groen RJ. Non-operative treatment of spontaneous spinal epidural hematomas: a review of the literature and a comparison with operative cases. Acta Neurochir (Wien) 2004;146(2):103-110
6 Cordova AC, Sumpio BE. Visceral artery aneurysms and pseudoaneurysms - Should they all be managed by endovascular techniques? Ann Vasc Dis 2013;6(4):687-693

7 Pulli R, Dorigo W, Troisi N, Pratesi G, Innocenti AA, Pratesi C. Surgical treatment of visceral artery aneurysms: A 25-year experience. J Vasc Surg 2008;48(2):334-342 